\title{
Arborescences
}

Revue d'études françaises

\section{Présence érudite et savoirs politiques dans la correspondance du Grand Condé}

\section{Guillaume Bazière}

Numéro 9, décembre 2019

La lettre érudite. Nouvelles recherches sur la communication savante à l'époque moderne ( $\mathrm{XVI}^{\mathrm{e}}$-XVIII ${ }^{\mathrm{e}}$ siècles)

URI : https://id.erudit.org/iderudit/1068274ar

DOI : https://doi.org/10.7202/1068274ar

Aller au sommaire du numéro

Éditeur(s)

Département d'études françaises, Université de Toronto

ISSN

1925-5357 (numérique)

Découvrir la revue

Citer cet article

Bazière, G. (2019). Présence érudite et savoirs politiques dans la correspondance du Grand Condé. Arborescences, (9), 49-67.

https://doi.org/10.7202/1068274ar
Résumé de l'article

Dans les travaux sur la « République des Lettres », la question de l'érudition et des savoirs est traditionnellement pensée de manière autonome par rapport aux problématiques sociales et politiques. Cet article analyse la présence d'acteurs possédant une légitimité savante dans la correspondance du Grand Condé (1621-1686) en considérant la lettre comme un véritable outil d'action politique et sociale. Cela permet de montrer, à travers l'étude de lettres comme celles de Pierre Michon Bourdelot, Bernardin Martin ou encore Isaac de Lapeyrère, comment la pratique des savoirs et l'action politique dans un contexte de service clientélaire sont intrinsèquement liées. Cela permet aussi d'identifier une police particulière d'usage des savoirs, qui définit un espace de communication efficace entre le prince et ses domestiques érudits.
Tous droits réservés @ Département d'études françaises, Université de Toronto, 2020
Coct protége par la loi sur le droit d'auteur. L’utilisation des services d’Érudit (y compris la reproduction) est assujettie à sa politique d'utilisation que vous pouvez consulter en ligne.

https://apropos.erudit.org/fr/usagers/politique-dutilisation/ 


\section{SOMMAIRE}

1 Sébastien Drouin, Université de Toronto

Camelia Sararu, Université de Toronto

Introduction

10 Myron McShane, Centre d'études sur la Réforme et la Renaissance, Université de Toronto Une lettre de Jean Dorat sur l'œuvre de Nonnos

31 Benoît Autiquet, Université de Bâle

Ce que la lettre familière fait au discours médical.

Une lecture de la lettre XIX, 16 des Lettres de Pasquier (1619)

49 Guillaume Bazière, Université Paris Nanterre

Présence érudite et savoirs politiques dans la correspondance du Grand Condé

68 Vanezia Pârlea, Université de Bucarest

Lettres d'Orient : échanges épistolaires en contexte interculturel dans les Mémoires du chevalier d'Arvieux

81 Yves Moreau, Université de Lyon

"Qualche novità litteraria»: la correspondance

entre Jacob Spon (1647-1685) et Antonio Magliabechi (1633-1714)

95 Corinne Marchal, Université de Franche-Comté

La dynamique de la circulation des savoirs et de leurs matériaux dans les échanges épistolaires entre Jean-Baptiste Boisot et Paul Pellisson-Fontanier (1674-1693)

106 Mathilde Chollet, Le Mans Université

Un «gai savoir»: stratégies du rire dans les lettres

$d$ 'une érudite des Lumières 


\title{
Présence érudite et savoirs politiques dans la correspondance du Grand Condé
}

\author{
Guillaume Bazière, Université Paris Nanterre
}

\section{Résumé}

Dans les travaux sur la "République des Lettres», la question de l'érudition et des savoirs est traditionnellement pensée de manière autonome par rapport aux problématiques sociales et politiques. Cet article analyse la présence d'acteurs possédant une légitimité savante dans la correspondance du Grand Condé (1621-1686) en considérant la lettre comme un véritable outil d'action politique et sociale. Cela permet de montrer, à travers l'étude de lettres comme celles de Pierre Michon Bourdelot, Bernardin Martin ou encore Isaac de Lapeyrère, comment la pratique des savoirs et l'action politique dans un contexte de service clientélaire sont intrinsèquement liées. Cela permet aussi d'identifier une police particulière d'usage des savoirs, qui définit un espace de communication efficace entre le prince et ses domestiques érudits.

Qu'observe-t-on lorsque l'on analyse la présence d'érudits dans la correspondance d'un acteur politique majeur? Quels types de savoirs et de pratiques apparaissent dans des lettres d'érudits échangées en dehors de la correspondance entre membres authentifiés de la "République des lettres»? Une étude récente (Cosandey 2016) a montré que nombre d'érudits de l'époque moderne sont au service de familles nobles, et ce, dans le cadre de stratégies développées autour de la défense du rang. Leur expertise dans de multiples domaines est, en effet, largement recherchée, tout comme leur capacité à drainer de l'information politique et diplomatique grâce à leurs larges réseaux de correspondants. Nous nous intéressons ici à la présence de l'érudition dans la correspondance, essentiellement passive $^{1}$, du prince du sang Louis II de Bourbon-Condé (1621-1686). Il s'agit donc de considérer les usages et la circulation de savoirs au sein d'un type d'écrits dont la forme et le fond sont fortement marqués par les caractéristiques du fonctionnement d'une maison princière à l'époque moderne (importance de l'information, notamment des nouvelles diplomatiques et militaires, de la question des finances, du divertissement mondain, du clientélisme, etc.). La prise en compte de ce contexte est cruciale. En effet, comme le soulignent J. Häseler et A. McKenna (2006: 8), « [p]our que l'analyse dépasse l'anecdotique, il faut cette mise en relation de la lettre avec la correspondance dans son ensemble comme aussi de l'individu avec la complexité des milieux sociaux qu'il fréquente». Plus largement, il s'agit donc de penser la communication comme un processus, ce qui «suppose l'attention aux pratiques et aux enjeux qu'elle recèle, aux stratégies mises en œuvre, aux usages [...]" (Beaurepaire 2014: 6). Cela nous amènera à tracer les contours d'un type original de discours épistolaire, travaillant les savoirs dans une visée d'efficacité pratique et de service, mais aussi à reconsidérer la figure de l'érudit en analysant ses lettres comme des actes permettant d'approcher l'identité plurielle du métier d'homme de savoir. Les acteurs étudiés ici ont tous à voir avec l'érudition au sens

1. Seules quelques rares minutes des lettres du Grand Condé sont conservées dans les Archives du Musée Condé à Chantilly. En ce qui concerne les cas traités dans le présent article, les éventuelles lettres envoyées par le prince n’ont pas été conservées ou ne sont pas connues. 
large que lui concède en 1680 le Dictionnaire françois de Pierre Richelet, en la définissant sobrement comme «savoir, doctrine, sience $[$ sic $]$ » (I: 300). Il ne s'agit pas de considérer la pratique d'une érudition classique tournée vers l'établissement de faits passés et déjà bien documentée (notamment Kriegel 1988 ; Grell 1993), mais d'examiner l'agir épistolaire d'acteurs qui soit jouissent de leur vivant d'une notoriété de docte (ainsi de Nicolas Malebranche), soit possèdent une légitimité savante de par leur profession et agissent d'une manière massivement reconnue et décrite dès le XVII ${ }^{\mathrm{e}}$ siècle comme celle du docte, de l'érudit, voire du pédant - figures qui renvoient à des comportements sociaux notamment érigés en types et caricaturés par la littérature du temps (Schlanger 1994; Roulier 2019). L'érudition est donc ici envisagée dans ses usages ordinaires dans la correspondance, sous la plume de savants de diverses conditions.

\section{Les correspondants du Grand Condé: un foyer d'érudition?}

La cour de Chantilly, foyer des princes de Condé, est réputée dans l'historiographie pour constituer un "pôle artistique indépendant» sous l'autorité de Louis II (Béguin 1999a: 329). Ce trait, qui apparaît comme singulier à une époque où la protection des artistes devient presque exclusivement l'apanage de l'État royal, semble s'étendre à la protection de savants et d'érudits. Le Grand Condé, réputé curieux de sciences, apparaît ainsi comme le défenseur des penseurs mis à l'index. Il est vrai que plusieurs figures rattachées par René Pintard à sa catégorie de «libertins érudits» (Pintard 1943²) peuplent la clientèle du prince, ainsi d'Isaac de Lapeyrère ou encore de Pierre Michon Bourdelot. La tenue de «l'académie Bourdelot» dans l'hôtel parisien du prince de Condé n'est d'ailleurs pas pour rien dans la réputation de celui-ci, considéré comme un protecteur des sciences. Pourtant, la caractérisation de l'activité de cette académie repose sur peu d'éléments, principalement sur les Conversations de l'académie de Monsieur l'abbé Bourdelot, publiées dans leur première mouture en 1672. Ce texte au statut ambigu, présenté comme un compte-rendu des séances en dépit d'évidentes marques de fictionnalisation, doit avant tout être considéré comme une production textuelle émanant de l'entourage de Bourdelot et non comme un authentique verbatim des réunions du cercle de curieux. Reste donc la correspondance du Grand Condé, peuplée de noms connus d'érudits, de savants, de penseurs, mais aussi d'inconnus dont la présence autour du prince est justifiée par leur savoir et leur expertise dans des domaines précis. Observer l'érudition par le biais de la correspondance d'un prince du sang, acteur politique majeur de son temps, permet de confronter une activité souvent saisie comme extraordinaire - le travail du savoir par une poignée d'hommes dont historiens et littéraires ont souvent admiré la soif de connaissances et la persévérance dans la recherche -, et cette activité éminemment ordinaire dans un lieu de pouvoir qu'est la correspondance, qui se caractérise par la régularité et la masse des envois, la présence répétée des mêmes correspondants et le peu de variété dans les contenus. C'est que la correspondance d'un prince qui se mêle de guerre et de politique est avant tout faite d'envoi de nouvelles, de transmission d'ordres et de consignes, de multiples comptes-rendus et de lettres flatteuses requérant un bienfait. Nous ne nous intéresserons donc pas à la question des pratiques scientifiques, discussions et autres expériences mises en œuvre par les savants et demi-savants gravitant autour du Grand Condé, mais à ce que l'on pourrait concevoir comme l'ordinaire de l'érudit dans sa correspondance, c'est-à-dire aux interactions entre légitimité savante et service du prince.

2. La catégorie de "libertinage érudit» a été discutée et critiquée de manière importante. Jean-Pierre Cavaillé y voit, avec raison, une catégorie «lourde aussi de présupposés qui brident le travail de réflexion historique», notamment en ce qu'elle induit une «histoire de la littérature et des idées séparées de l'histoire sociale» (Cavaillé 2011). 
Une première recension des correspondants du Grand Condé possédant une légitimité savante semble dévoiler une organisation au sein de laquelle des acteurs intégrés dans des réseaux érudits cultiveraient de manière extraréticulaire un lien épistolaire avec le prince. Les acteurs en question seraient alors définis avant tout par leur identité érudite et la pratique d'activités savantes (comme la tenue d'académies ou la correspondance avec d'autres savants notoires). Dans cette configuration, le lien avec le prince n'apparaît qu'à la marge: le prince protège, encourage, questionne, s'intéresse et se tient éventuellement au courant des discussions agitant une nébuleuse savante. Celle-ci semble présenter une certaine autonomie et ses interactions avec certains lieux de pouvoir permettent de définir des puissants comme amis des sciences ou encore des arts. Il s'agit là de l'idée qu'il existerait d'une part une "République des Lettres" plus ou moins autonome et affranchie du politique, et d'autre part des lieux de pouvoir, éventuellement intéressés par le savoir, mais réglés par des rapports de force sociaux, politiques, diplomatiques ou encore militaires ${ }^{3}$. Cette conception a déjà été fortement battue en brèche. L'analyse de la carrière de Valentin Conrart a ainsi permis de démontrer que l'on ne peut "considérer la "république des lettres" comme un monde clos, replié sur les espaces de l'érudition et de l'échange entre savants", puisque "[l]a spécificité du professionnel des lettres du XVII ${ }^{\mathrm{e}}$ siècle réside dans un certain nombre de compétences propres, mais qui sont investies dans des actions dont les enjeux, sociaux et politiques dépassent de loin l'échange érudit» (Schapira 2003: 275-276). L'analyse de la position sociale des correspondants du Grand Condé présentant une légitimité savante abonde en ce sens, puisque nombre d'entre eux se révèlent être au centre de l'organisation de la maison princière. Leur espace d'action est donc éminemment politique.

Quelques rares exemples de discours prenant l'aspect caractéristique de dissertations érudites peuvent être relevés dans la correspondance du Grand Condé. C'est notamment le cas pour la première lettre de Bernardin Martin, un des apothicaires du prince ${ }^{4}$, qui devient un correspondant régulier à partir de 1673. Martin est né le 8 janvier 1629 à Paris. Il est le fils de Samuel Martin, un «apothicaire et distillateur" protestant ${ }^{5}$ attaché au service de Marie de Médicis ${ }^{6}$. Il entre au service du prince de Condé au tout début des années $1670^{7}$. On ne sait à ce jour quasiment rien des quarante premières années de sa vie. C. Gysel (1980: 61) émet toutefois l'hypothèse qu'il ait étudié à Montpellier. Le $1^{\mathrm{er}}$ juin 1673, il envoie au Grand Condé une lettre, la première conservée dans les archives parmi les nombreuses missives envoyées par l'apothicaire à son illustre patron:

Puisque vostre altresse Sérénissime veult bien me faire l'honneur de souffrir que je luy escrive, \& qu'elle a la bonté d'escouter ceux qui tachent de s'advencer dans la cognoissence des secrets de la nature, je prendray la liberté de l'entretenir de quelques expériences qui, moyennant sa faveur, ont esté faictes dedans mon laboratoire depuis son départ. Nous avons anatomisé le vin comme l'aliment le plus commun à l'homme $\&$ qui mesme luy est d'un usage adventageux lorsque est pris en modeste quantité et ajusté au tempérament de la personne. Il contient des esprits ${ }^{8}$ qui aydent à la circulation

3. Cette théorie se retrouve notamment dans les travaux de Marc Fumaroli (2015).

4. Sur le statut d'apothicaire à l'époque moderne, voir la mise au point d'Alexandre Lunel (2008: 25-30).

5. Sur les apothicaires protestants à l'époque moderne, voir Claude Cabanis (1987).

6. Voir l'Inventaire après décès de Pierre Boulle dressé le 27 septembre 1635, où Samuel Martin est cité comme témoin.

7. Dans l'épître dédicatoire de son Traité de l'usage du lait (1684), il affirme être au service du Grand Condé depuis quinze ans (ã iij $\mathrm{r}^{\circ}$ ), ce qui situerait son entrée dans la maison princière en 1669. Les premières lettres de Martin n'apparaissent toutefois qu'en 1673.

8. «En general les Chymistes appellent esprits, les petites parties non aqueuses qui s’élevent des corps par la chaleur, \& se reduisent en liqueur par la distillation» (Furetière, Dictionnaire universel, article «Esprit»). 
du sang comme je le feray veoir cy après à V. A. S. lorsque je lui parleray des espreuves que nous avons faicts sur le sang. Je ne m'amuseray pas à informer V. A. S. de toutes les questions qui se sont faictes dans notre assemblée sur ce chap[itre] comme sur beaucoup d'autres, parce que ce seroit fatiguer V. A. Je m'attacheray à ce qui m’a paru le plus utile \& luy diray seulement que tirant moins d'esprits des gros vins rouges et forts, il est moins favorable à la santé de la pluspart des hommes que ceux qui sont $[\ldots]$ paillets ${ }^{9}$, parce que ceux-là abondent en plus grande quantité du tartre, qui retient et fixe en quelques façon les esprits, au lieu que ceux cy, subtilisant les humeurs, les rendent plus flexibles \& coulantes, et comme il faut de la modération en toutes choses, il est aussy dangereux de les choisir trop subtiles parce qu'à succession de temps, abimant trop les humeurs, il se faict une demesurée dissipation d'esprits qui enlevent et entrainent avec eux dans les petites conduites du cerveau des parties terrestres qui venant à les boucher causent des apoplexies et paralysies que nous voyons assez fréquament arriver à ces amateurs de vins si délicats [...]. Ce texte [sic] donna occasion à quelques uns de parler du vinaigre et de demander comment cela ce faisoit. L'on convaint qu'il ne se faisoit que par la dissolution du tartre, laquelle se fait lorsque quelques quantités d'esprits les plus subtiles se sont évaporés, \& pour preuve que c'est le tartre qui faict l'acide, c'est que l'on ne trouve presque point de lie dans le vinaigre, $\&$ nous avons tiré un sel semblable au sel de tartre, $\&$ ayant fait sécher le caput mortuum ${ }^{10}$ à feu lent, l'acide que l'on a jeté dessus l'a faict bouillonné et comme je n'estois pas satisfait sur les alkalys ${ }^{11}$ ou sels fixes, sur quoy V. A. eut la bonté de raisonner une fois à Chantilly, je recommençais à agiter la chose sur l'effervescence que les acides font sur les alkalys. Il se trouva un parti qui soutint que c'estoit les sels fixes qui tuoient les acides et qui les fesoient agir, \& que comme il y avoit un sel universel en toutes choses, le bouillonnement par l'acide s'en fesoit plus ou moins évident. Mais l'expérience nous a faict voir le contraire. [...]. (P 47 : fo 3-4)

Bernardin Martin décrit alors les différentes expériences menées pour prouver que ce ne sont pas les sels qui font agir les acides. Il rapporte ensuite l'intervention d'une nouvelle personne qui relance le débat:

Ces expériences estant faites, une personne capable, mais plus abondant en son sens que les autres dit qu'il n'y avoit de sel universel que celuy qui se trouvoit dans les plantes et dans les pierres. Cependant, il y a bien plus d'apparence que le sel le plus pur de tous faict par la nature \& qui se trouve partout en plus grande quantité comme est le sel marin et son semblable, soit plus universel que celuy qui a esté tiré d'une plante par le feu. Cette mesme personne adjouta qu'il pouvoit avoir dans la chaux un sel qui fesoit l'effervescence, mais pour le satisfaire, nous luy laissames tirer tout le sel qu'il prestendoit estre dans la chaux $\&$ après l'avoir desseiché nous y versames de l'acide qui fit son bouillonnement, comme si tout le sel qui y estoit y eut esté encore. (P 47: fo 5)

Il passe ensuite à la description de plusieurs expériences sur le sang humain, avant d'achever ainsi :

Voilà, Monseigneur, de quoi bien occuper les philosophes de votre maison si la bravoure des Hollandois n'a pas augmenté depuis l'année passée, on m’a dict qu'ils estoient incorrigibles sur le Proverbe \& que leurs vêtements rouges ne leurs avoient pas réussy, peut-estre aussy n'ont-ils pas ouy dire que l'habit ne fait le moine. (P 47: fo 6)

9. «Epithete qu'on donne particulierement au vin, \& aux liqueurs qui devroient estre rouges, \& qui sont neanmoins pâles \& claires» (ibid, article "Paillet»).

10. Désigne un résidu dont on ne peut rien extraire.

11. "C'est un sel vuide \& poreux disposé à se joindre facilement à tous les acides» (Furetière, Dictionnaire universel, article «Alkali»). 
La lettre se conclut par de courtes nouvelles de la santé des petits-enfants du Grand Condé. Prise dans son ensemble, elle apparaît comme un modèle de lettre érudite. En effet, elle est écrite par un apothicaire, qui, même s'il ne passe pas à la postérité, puisqu'il peine à être identifié dans les grands dictionnaires biographiques ${ }^{12}$ et n'apparaît pas dans l'historiographie sur la "République des Lettres", parvient en son temps à publier quelques ouvrages ayant trait à la médecine dont une Dissertation sur les dents (1679) et un Traité de l'usage du lait (1684). Si la dimension pratique du métier d'apothicaire au XVII ${ }^{\mathrm{e}}$ siècle est indéniable (Lafont et Van Robaeys 1992), les écrits publiés de Bernardin Martin, notamment celui sur les dents de 1679, montrent bien qu'il manie un savoir qui, loin de reposer uniquement sur une pratique technique, voire expérimentale, est le fruit de la sédimentation de connaissances issues de la tradition médicale aussi bien orale que livresque (Gysel 1980: 63-64; Ruel-Kellermann 2010). La pratique érudite des savoirs, et en particulier des sciences, n'est d'ailleurs pas étrangère à l'élite des apothicaires à l'époque moderne, comme l'a démontré Olivier Lafont (2008: 38-39 et 87-90), mettant en lumière la carrière de l'apothicaire Matthieu-François Geoffroy et de ses descendants. Bernardin Martin est d'ailleurs un des participants aux réunions de l'Académie Bourdelot (Allaire 1886: 137-138). Et, en effet, la lettre envoyée au prince n'en finit pas, se perd dans les détails, les digressions, fonctionne par association (le vin, le vinaigre, le sang), décrit des expériences, utilise des termes associés dans les dictionnaires du temps à des domaines restreints du savoir comme la chimie ou la physique. Elle évoque un débat à plusieurs voix, où chacun avance ses hypothèses dans une forme dialoguée courante au XVII ${ }^{\mathrm{e}}$ siècle et qui, inspirée entre autres par le modèle platonicien, constitue «le véhicule littéraire le plus apte à représenter le débat d'idées» (Pujol 2005: 3). Elle est enfin adressée à un interlocuteur qui est décrit comme disposé à accueillir les propos de ceux qui se piquent de percer les "secrets de la nature». Nouvellement arrivé dans la maison princière, Bernardin Martin veut faire valoir son savoir et ses compétences de chimiste auprès du prince, sa connaissance des éléments et son habileté à mener des expériences. Même si une part du savoir utilisé présente un caractère expérimental, l'usage qui en est fait relève bien plutôt de l'érudition en ce qu'il s'agit d'un savoir qui ne vient pas nourrir une démarche scientifique pratique, mais qui est réinvesti dans une écriture épistolaire caractérisée par l'accumulation et l'énonciation minutieuse de données savantes ${ }^{13}$. La lettre est tout à la fois lettre savante, frisant le pédantisme, lettre de louange d'un prince décrit comme ami des sciences et lettre de gratitude. Martin précise que les expériences sont possibles grâce à la faveur du prince qui abrite des "philosophes" en sa maison, catégorie que Richelet, dans son dictionnaire de 1680, définit comme ceux «qui sa [vent], qui croi[en]t savoir, ou qui se pique[nt] de savoir la Logique, la Morale \&

12. Il n'apparait par exemple pas dans Le grand dictionnaire historique de Louis Moreri, tandis que l'on y trouve bien Pierre Michon Bourdelot (dès l'édition de 1718) ou encore Isaac de Lapeyrère. Dans le Dictionnaire historique et critique (1697) de Pierre Bayle, il ne dispose pas de notice dédiée mais apparaitt en vertu d'une digression présente dans l'article sur le médecin néerlandais Van der Linden. En effet, Bayle explique que, bien que l'ouvrage bio-bibliographique De scriptis Medicis (1637) de Van der Linden ait été augmenté par le médecin allemand Merklinus et republié en 1686, il reste toutefois incomplet. Il donne alors en exemple l'absence de Bernardin Martin et fournit à cette occasion une courte notice biographique sur l'apothicaire (Bayle, Dictionnaire historique et critique, II: 1206). C. Gysel (1980 : 59) cite plusieurs dictionnaires des XVIII et XIX ${ }^{\mathrm{e}}$ siècles qui transforment son prénom et le confondent avec une autre personne. Panckoucke le mentionne sans erreur dans le tome 6 de sa Biographie médicale en 1824 et le qualifie de "chimiste» (203). Bernardin Martin n'est donc pas tout à fait oublié, mais se retrouve cantonné à des dictionnaires spécialisés sur la médecine.

13. La «construction d'un discours et d'un récit» à partir de grandes quantités de données brutes tout comme la «transmission des savoirs" sont bien des éléments caractéristiques d'un travail érudit, comme le rappelle François Brizay (2015: 12). 
la Phisique» (Dictionnaire françois, II : 157). Martin veut attiser la curiosité du prince et semble s'attendre à ce qu'il lui en demande plus. Il précise en plusieurs endroits qu'il ne dit pas tout, qu'il ne veut pas ici ennuyer le prince, mais pourrait donner une tout autre ampleur à sa dissertation. Il sait se mettre en avant: la polyphonie du compte-rendu est faussée. Martin, en position de narrateur, se réserve ainsi le rôle du sage savant, tandis qu'une "personne» s'égare dans l'erreur et se le voit démontrer par un Martin pédagogue qui le laisse mener ses expériences vouées à l'échec "pour le satisfaire». Cette lettre est bien une lettre savante, mais qui n'a rien d'un écrit circulant dans un espace où seul compte l'échange désintéressé de connaissances. Il y a bien une logique d'échange, mais celui-ci s'opère sous le régime comptable du don et du contre-don. Bernardin Martin offre au prince non pas seulement des connaissances, mais aussi un éloge qui ne s'énonce pas directement. En effet, si l'hyperbole laudative est absente de la lettre, la louange est présente à travers l'exposé de ce que permet la bienveillance du prince envers les "philosophes» qui peuplent sa maison. En échange, l'apothicaire attend l'expression de la satisfaction du prince et l'octroi de moyens matériels permettant de persévérer dans sa voie. Il annonce même à la fin de la lettre l'envoi prochain d'une petite pièce d'écriture de sa main au prince, autre forme de présent d'un savant lettré à un Grand:

Je ne dis encore rien à V. A. des Beautés de Chantilly parce que il y a si peu que j’y suis que si Phobus ne m'abandonne, j'espère avec un peu de temps vous faire une relation à ma mode, si je suis assez heureux pour qu'elle plaise à votre altesse, je serai le plus content de la terre, car de bonne foy, je ne croye pas qu'il y aye personne au monde qui soit plus animé à s'acquitter de son devoir envers $\mathrm{V}$. A. [...] (P 47: fo 6)

Le geste de Bernardin Martin s'inscrit donc dans une logique de mécénat, qui fonctionne selon un principe de «reconnaissance mutuelle» du savant ou «de l'artiste et du Grand» (Viala 1985: 54). Or, Condé n'est pas le mécène de Bernardin Martin. Ce dernier est un domestique ${ }^{14}$ de sa maison et, à ce titre, sa position relève d'une logique de service. Et en effet, la lettre de Bernardin Martin est un coup d'épée dans l'eau. Il semble s'être fourvoyé sur son rôle auprès du prince. Sur les 91 lettres que Bernardin Martin envoie au Grand Condé entre 1673 et 1686, elle est en effet la seule de ce type. Ses autres lettres ne font plus étalage d'érudition, mais consistent en des comptes-rendus médicaux sur la famille du prince, accompagnés d'informations plus générales d'ordre politique, diplomatique, militaire et de nouvelles de la cour. Il est fort probable que le rôle de Martin dans la maison du prince lui ait été explicité après l'envoi de ce savant morceau de bravoure. Il s'agit donc d'une entrée en communication ratée, prélude à la construction d'un espace de communication qui sera finalement défini selon les logiques de fonctionnement de la maison princière. Un indice supplémentaire confirme le malentendu initial sur ce que doit être le contenu des lettres adressées au prince. En effet, les lettres de la correspondance des princes de Condé sont souvent munies d'un résumé en plusieurs points inscrits au dos du dernier feuillet. Cette pratique de secrétaires permet avant tout un archivage efficace, mais peut aussi être utilisée par le prince pour identifier rapidement l'intérêt d'une lettre ou en retrouver les informations essentielles. En ce qui concerne les lettres de Bernardin Martin portant sur la santé de la famille du prince, le résumé est souvent relativement détaillé, reprenant les principaux points soulevés par l'apothicaire. Ici,

14. On rappelle qu'au XVII ${ }^{\mathrm{e}}$ siècle, le terme de "domestique» appliqué à ceux qui appartiennent à une "maison" (une famille, souvent illustre) désigne ceux qui assurent fidélité et service auprès d'un maître contre protection et revenus. L'acception est large et l'on peut très bien être noble et domestique. Les domestiques, dans leur diversité, constituent ainsi un vivier d'agents politiques et d'hommes de confiance servant à diverses missions. La domesticité, en particulier chez les Grands, relève ainsi plus de logiques de clientèle et de fidélité que du salariat. 
sur cette lettre du $1^{\text {er }}$ juin 1673, la partie sur les expériences, qui occupe 136 lignes sur un total de 142, ne fait l'objet que de la mention laconique suivante: "raisonne sur quantité d'expériences qu'il allegue ». Aucune mention n'est donc faite de la forme que prennent ces expériences, ni même simplement de leur sujet. Et pour cause, la lettre s'épuise dans une description qui n'est d'aucune utilité pour le service du prince, puisqu' elle sert avant tout à mettre sous le regard de celui-ci les pratiques de Bernardin Martin et de ses acolytes. La description est donc à elle-même sa propre fin. Or, la correspondance est pour Condé, comme pour tout acteur politique, avant tout un outil d'action. La leçon semble avoir été bien retenue puisque dans une lettre du 16 décembre 1682, Martin expose à Condé un remède contre la goutte dont souffre le prince. Il décrit en détail le régime qui doit l'accompagner et donne l'exemple d'une personne sur laquelle l'ensemble réussit parfaitement. Il ajoute enfin: "Si je ne craignois pas de sortir de mon caractère, je pourrois appuyer cette méthode de raisons qui parraitroient valables pour estre persuadé que cela ne pourroit, Monseigneur, estre nuisible» (P 88: fo 275). L'affirmation sert à convaincre le prince, mais Martin se garde bien de rentrer dans les détails de ses «raisons» et contient sa plume qui pourrait facilement déborder de son rôle si elle s'égarait dans des démonstrations retorses. Le savoir est nécessaire au service du prince, mais se doit d'être contenu dans d'efficaces limites.

Une autre forme de présence érudite dans la correspondance Condé est celle signalée par l'apparition d'un expéditeur connu pour son savoir. C'est par exemple le cas de l'oratorien Nicolas Malebranche, qui se fait connaître comme théologien et philosophe avec la publication de son traité De la recherche de la vérité, dont le premier tome paraît en 1674, et qui est élu en 1699 à l'Académie des Sciences. Dans une lettre de 1681, le prêtre de l'Oratoire écrit à Condé pour lui envoyer un exemplaire de De la recherche de la vérité "plus commode et quelque peu plus correct que celui dont vous vous servez" (P 81: fo 329). Il pourrait s'agir de la quatrième édition de 1678 publiée en un volume in $-4^{\circ}$ chez André Pralard à Paris. La suite de la lettre montre que Malebranche recherche en fait le soutien du prince au sujet de son récent Traité de la nature et de la grâce, paru en 1680. Il explique succinctement au prince les raisons de certains de ses choix de composition, et notamment l'absence de citations de saint Augustin dans ce nouvel ouvrage. De nombreuses critiques, à cette époque, visent en effet l'oratorien et lui reprochent de ne pas se conformer aux dogmes augustiniens sur la grâce. Katia Béguin note d'ailleurs avec justesse que «l'oratorien quêtait moins une défense contre la censure [...] qu'une approbation dans la polémique qui l'opposait à Bossuet, Arnauld ou Fénelon, depuis la parution [...] de son Traité» (Béguin 1999a: 376). Le Grand Condé semble toutefois effectivement intéressé par le théologien et ses écrits. Malebranche est d'ailleurs reçu à Chantilly. En effet, une lettre du 18 août 1683 qu'il écrit à Pierre Berrand nous apprend que le philosophe a demeuré «deux ou trois jours » à Chantilly à la demande de Condé (Malebranche, Euvres complètes: 256). Malebranche explique que le prince a souhaité le rencontrer après avoir lu De la recherche de la vérité qu'il apprécie beaucoup. Il insiste sur "l'esprit vif» de celui-ci et sur la qualité de sa conversation. L'intérêt du prince pour la fréquentation de Nicolas Malebranche doit toutefois être mis en rapport avec la religiosité nouvelle qui semble caractériser le Grand Condé sur la fin de sa vie. Il s'agit donc avant tout d'intérêts communs entre un prince qui apprécie la lecture et se rapproche de Dieu et le théologien cherchant soutien et oreille attentive. La teneur des discussions reste privée et rien n'indique qu'il ait existé des échanges entre Condé et Malebranche sur le fond des écrits de ce dernier. Les apports réciproques de cette entente entre le prince et l'oratorien semblent tenir davantage de l'entretien entre le directeur de conscience et l'homme revenant sur les chemins de la foi que du débat d'idées entre lettrés. Condé meurt trois ans plus tard et l'on ne connait aucune trace d'un soutien effectif du prince au philosophe, ni d'échanges suivis et fertiles entre les deux hommes. 
Il existe pourtant bien, conservés avec la correspondance, des écrits consacrés en totalité à des questions de science. Il s'agit essentiellement de nouvelles portant sur des actualités scientifiques. Elles sont rédigées le plus souvent à la manière de mémoires et transmises en parallèle des lettres. Il en est ainsi d'un mémoire joint à une lettre de Bourdelot du 17 mai 1679 (P 73 : fo 244). Le mémoire évoque une rencontre entre Bourdelot et l'abbé François de Lanion au cours de laquelle ils ont discuté de la loi de la réflexion mise en évidence par Descartes. Bourdelot rapporte les raisons de l'abbé (qui s'oppose au philosophe tourangeau), puis évoque le point de vue d' "autres» personnes. Il annonce enfin la publication prochaine de deux livres de Christian Huygens sur les lois de la réfraction. L'ensemble occupe une trentaine de lignes sur deux feuillets de format réduit $(21,5 \times 18 \mathrm{~cm})$. Il ne s'agit donc pas d'un exposé détaillé visant à donner au prince les moyens intellectuels de s'immiscer dans le débat, mais plutôt d'une efficace et brève mise au point sur les différentes théories en vogue à la fin des années 1670 sur le sujet de la lumière. Dans les années 1677 et 1678, Huygens rédige en effet son Traité de la lumière (qui ne sera publié qu'en 1690). Il discute alors ses vues avec plusieurs correspondants et informe le ministre Colbert de ses découvertes (Chareix 2006: 122). Les résultats de ses travaux sont ainsi connus aussi bien parmi les curieux des sciences que dans les hautes sphères de l'État. À la différence des expériences de Bernardin Martin, la question de l'optique constitue donc un sujet en vogue, dont la connaissance peut s'avérer profitable au prince, que ce soit par goût de se tenir informé de ces matières ou par souci de tenir son rang en société. Le format du mémoire, rédigé à part, est d'ailleurs le témoin d'une forme d'utilisation différente de celle de la lettre, qui est quant à elle plutôt tournée vers l'action immédiate, l'échange et la personne du prince. Le mémoire sur des questions de science, soigneusement travaillé et mis en forme, peut être ignoré, archivé à part (ce qui n'est pas le cas ici), ou encore diffusé, prêté, montré, sans que le contenu d'une lettre où peuvent apparaître des propos plus intimes ou confidentiels soit dévoilé. Loin d'être adressé à un seul destinataire, ce type de texte fait d'ailleurs souvent l'objet de multiples envois, y compris en dehors de la maison princière.

Ainsi, s'il y a bien présence d'acteurs possédant une légitimité savante et si des usages des savoirs sont bien en jeu, la correspondance princière n'apparaît en rien comme un espace qui serait, pour une part, dédié exclusivement à la discussion, à l'accroissement et à l'échange désintéressé de connaissances dans une perspective humaniste. J. Boutier, après avoir noté que la conception de la lettre comme "outil pour l'action" apparaît dans les manuels de correspondance de l'époque moderne comme le Segretario de Francesco Sansovino (1565), affirme d'ailleurs bien avec justesse que les "lettres savantes" et les "écritures les plus ordinaires" "apparaissent [...] comme un ensemble de documents indissociables dès lors que l'interrogation historienne les envisage comme des moyens essentiels aux conduites de l'action politique» (2009: 11). Il s'agit donc d'analyser ici comment s'articulent usages des savoirs et logiques de service en un lieu politique et comment cette articulation se traduit dans le discours épistolaire au prince.

\section{Logiques politiques et présence ordinaire du savoir dans la correspondance}

La recherche de traces d'érudition pour illustrer la présence d'une activité érudite autour du prince ne saurait aboutir qu'à un résultat tautologique qui ferait fi des logiques sociales à l'œuvre dans un lieu tel que la maison d'un prince du sang. Il s'agit donc ici de recontextualiser les différents éléments en prenant pleinement en compte ce lieu d'énonciation singulier qu'est la correspondance princière. 
Beaucoup des correspondants du Grand Condé possédant une légitimité savante ont un rôle lié au domaine de la médecine: c'est le cas de Bernardin Martin, de Pierre Michon Bourdelot, mais encore de Louis Bouillet et de Jean-Baptiste Dodart, deux autres médecins du prince. La plupart des lettres de Bourdelot et de Bernardin Martin sont d'ailleurs consacrées à des comptes-rendus médicaux sur la santé de la famille du prince. Pierre Michon Bourdelot, né en 1610, est le fils d'un chirurgien qui a lui-même exercé dans la maison des princes Condé. Il travaille d'abord comme médecin de l'ambassadeur François de Noailles, avant d'entrer à la fin des années 1630 au service d'Henri II de Bourbon, alors gouverneur de sa province natale, la Bourgogne. La carrière de Bourdelot s'inscrit donc dans un attachement ancien à la lignée des princes de Condé. Cette proximité n’est véritablement interrompue qu'entre la Fronde et le retour de Condé d'exil en 1659, puisque le médecin est prudemment parti se mettre au service de la reine Christine de Suède dès 1651 (Béguin 1999a: 405). Le retour dans le giron princier n'a rien de surprenant puisque l'obtention d'une place privilégiée dans la maison et le maintien dans les bonnes faveurs du prince sont un avantage certain pour Bourdelot, qui peut ainsi «se libérer des obligations ordinaires du praticien" (Béguin 1999 b: 86). "Quémandeur impénitent» (Béguin 1999 b: 87), le médecin voit ses revenus exploser dès la fin des années 1660 par le fait du prince qui lui donne notamment deux seigneuries en 1669. Les actes de donation stipulent bien que ce sont les bons soins du médecin qui sont récompensés (Béguin 1999 b : 87). Or, la défense et illustration de la qualité du travail du praticien passe notamment par l'écriture de nombreuses lettres au Grand Condé. Souvent, l'énonciation épistolaire du diagnostic est l'occasion pour le médecin de faire la démonstration de l'étendue de son savoir. Ainsi, le 22 mai 1665, Bourdelot écrit une lettre au prince qui vient de l'informer de la reprise de ses crises de goutte:

Je ne croy pas que le mal dure car vous n'avez pas de plénitude ${ }^{15}$. Je ne puis imputer ce prompt retour de douleur qu'à ce que votre altesse ne demeura pas en repos le jour de son purgatif, car, par l'agitation, les humeurs vont à l'habitude du corps et par l'application de l'esprit elles montent à la teste et retombent dans leurs poches de la ratte où elles demeurent, les intestins n'estant plus irrités par le purgatif. V. A. s'est agitée trop tost après. Elle alla à la chasse à Chantilly $\&$ à Valery, \& dans le printemps que les humeurs bouillent, je comprends comme elles se sont portées au pied. Il faudra doresnavant estre plus precautioné [...]. La chute de quelques humeurs a causé la douleur, mais il n'y a point de conspiration des grands vaisseaux \& cette attaque apparemment sera sans fiebvre. M. Bouillet aura vu s'il y aura eu élévation au poux $\&$ quelques réplétion $\&$ n’aura pas manqué à vous proposer la saignée. Quand les corps se remplissent de chairs, que la nourriture profite [...] les saignées sont nécessaires \& souvent des lavements après la cessation de la douleur. (P 33: fo 100)

Bourdelot est ici pleinement dans son rôle de médecin ordinaire, c'est-à-dire de domestique au service du prince. Seulement, en cette fin de mai 1665, le praticien, qui se trouve à Paris, n'est pas en mesure d'examiner Louis II, qui, le 20 mai, se trouvait à Dijon, dans son gouvernement de Bourgogne ${ }^{16}$. La distance n'empêche toutefois pas le médecin de suivre l'évolution de la santé de son prestigieux patient par le biais de la correspondance. Dans une lettre du 16 mai, le prince informe Bourdelot de ses douleurs liées à la goutte ${ }^{17}$. Le médecin est alors en mesure d'expliquer l'apparition de la crise à partir de l'analyse de l'historique des traitements administrés au prince et de ses activités.

15. Terme de médecine hippocratique désignant l'abondance du sang et des humeurs, corrigée le plus souvent par la purgation et la saignée.

16. Lettre du prince de Condé à M. Roy le 20 mai 1665, de Dijon (P 33: fo 68).

17. La lettre n'est pas conservée, mais Bourdelot l'évoque au tout début de sa lettre du 22 mai. 
La lettre du 22 mai est particulièrement intéressante, car elle montre l'intrication fine du devoir de service de Bourdelot, de la nécessité de se montrer efficace, voire indispensable, et de l'usage social, non seulement des savoirs, mais aussi de la position d'autorité conférée par une réputation d'érudit ou de savant. La position de Bourdelot n'est en effet pas si ferme qu'elle pourrait sembler l'être à la lumière déformante de sa postérité d'érudit libertin. Dans une lettre de 1659 (P 22: fo 147), où il se plaint d'être persécuté par ses adversaires et déplore que la reine Christine de Suède ne soit pas assez puissante pour le protéger, il demande à revenir sous la protection et au service du Grand Condé, alors que ce dernier revient en France après son exil consécutif à la Fronde. Le prince accepte, mais, malgré la bienveillance de ce dernier, Bourdelot est régulièrement contrarié dans ses prescriptions, notamment par les refus de la princesse de Condé, hostile à ses fréquents recours à des sirops purgatifs ${ }^{18}$. Il doit également composer avec d'autres domestiques du prince, principalement Louis Bouillet et Nicolas Languereau, respectivement médecin ordinaire et premier chirurgien du prince. Il s'en plaint d'ailleurs dans une lettre faisant suite à celle du 25 mai et écrite alors que la crise de goutte de Condé s'est aggravée: "J'auray à faire quelques reproches à $\mathrm{M}^{\mathrm{rs}}$. Bouillet \& Langros [sic] qui ne m'escrivent jamais sur tout dans une affaire importante comme celle cy» (P 33: fo $109 \mathrm{v}^{\mathrm{o}}$ ). La distance entre le prince et Bourdelot peut permettre aux deux autres praticiens de prendre le pas sur leur confrère et leur évite de se trouver en situation de subordination en devant obéir aux prescriptions de ce dernier. Pour maintenir sa position, Bourdelot n'a d'autres moyens que la plume et le papier. Ne pouvant démontrer son degré de savoir et d'expertise par l'exercice de son art auprès du prince et sur son corps même, il lui transmet une analyse de sa maladie qui ne vise pas à soigner (aucun remède n'est proposé dans la lettre), mais qui permet à Bourdelot de circonvenir la maladie du prince en convertissant l'irruption soudaine et inquiétante de la crise en un discours retraçant l'archéologie physiologique de cette affection. Bourdelot ne dispose en fait que de très peu d'éléments pour construire ce discours rationnel: une purgation (pratique ordinaire et fréquente), une partie de chasse (qui l'est tout autant) et enfin un contexte temporel : le printemps qui met les humeurs en ébullition. Le chemin à parcourir entre ce fait anodin dont dispose notre médecin mal informé (la purgation) et la manifestation de la crise de goutte n'est qu'affaire de logique et d'érudition. Pourquoi d'érudition? Le terme est ici intéressant si on le prend dans son sens le plus courant au XVII ${ }^{\mathrm{e}}$ siècle: celui d'un savoir de docte, déconnecté de la pratique et souvent associé à la pédanterie ${ }^{19}$. La démonstration logique construite par Bourdelot n'a aucune visée médicale pratique (elle n'appelle aucune manipulation particulière), ni ne cherche à faire progresser la science (elle se fonde simplement sur la très classique théorie des humeurs). Elle a donc bien un lien avec l'érudition en tant qu'elle mobilise une doctrine, mais ne permet pas ou ne vise pas l'expérimentation ou la découverte. Elle n'est toutefois pas érudition, mais mobilise plutôt celle-ci en vue de réaliser une action: prouver au prince que même à distance, Bourdelot reste indispensable, et cela malgré la présence à ses côtés de Bouillet et de Languereau. Le savoir exhibé et la virtuosité à en user ne servent toutefois pas directement à glorifier le médecin. Ils servent avant tout à rassurer, ils ont une fonction lénifiante: la cause est connue, le mal ne saurait durer. Bourdelot s'attache d'ailleurs, dans la lettre suivante du 25 mai (P 33: fo 109 vo), à rassurer le prince en démentant le caractère intraitable de sa goutte, hypothèse que le prince avait évoquée en expliquant la progression du mal vers les genoux. Bourdelot argumente encore là avec la théorie des humeurs, expliquant savamment la migration des maux. Abonder dans le sens de l'incurabilité de la maladie reviendrait à admettre sa propre inutilité.

18. Bourdelot s'en plaint par exemple dans une lettre du 12 septembre 1663 alors qu'il se trouve à Bourbon l'Archambault, accompagnant la princesse qui prend les eaux (P 29: fo 404).

19. C'est par exemple le sens qui ressort dans le Dictionnaire universel de Furetière. 
L'inverse lui permet au contraire de proposer divers remèdes, ce qu'il s'empresse de faire dans la lettre du 25 mai. La lettre du 22 mai porte ainsi une érudition singulière. Elle ne vise ni à faire preuve d'une érudition massive (la démonstration de Bourdelot n'est nourrie d'aucune référence savante, d'aucune sentence latine ou grecque), ni à faire circuler un savoir, non plus qu’à instruire. Elle est une érudition réinvestie socialement dans l'action: le prince est malade, le médecin doit agir. Cette érudition singulière est ici déterminée par le lieu de son énonciation: la lettre d'un médecin pourvu d'une légitimité savante, adressée à un prince du sang dont les immédiates préoccupations sont de nature politique, diplomatique et militaire. Il y a donc bien présence de l'érudition dans ce lieu éminemment politique qu'est la correspondance du prince, mais elle apparaît de manière bien particulière, en étant utilisée en tant que matière pour l'action dans le champ social. Bourdelot maintient et justifie sa position par une utilisation adaptée d'une érudition convertie en un discours rassurant pour le prince, tandis que Condé semble avoir foi dans la capacité de son médecin à le soulager, puisque même éloigné, il s'en remet à lui.

Un autre avatar de la présence de savoirs érudits dans la correspondance du Grand Condé consiste en la relation de curiosités. Ainsi, le 17 septembre 1682, Bourdelot écrit une lettre au prince de Condé dans laquelle il commence par évoquer la santé de la princesse Palatine, avant de fournir diverses nouvelles parmi lesquelles l'affaire d'un «relaps huguenot» et les dernières tractations diplomatiques avec l'Angleterre. Il conclut enfin par une curieuse information:

$\mathrm{M}^{\mathrm{rs}}$. Defita et Driancourt este à Charenton où une fille vomit des crapauds, des araignées et des laizards. Elle a de grandes douleurs. Elle accuse une vieille femme de luy avoir donné un sort. Le Roy la dit hier matin à $\mathrm{M}^{\mathrm{r}}$. De la Renye [sic] qui m’y doit mener. (P 87: fo 208)

On apprend finalement dans une lettre du 20 septembre que Bourdelot n'a pu se rendre auprès de la victime de l'ensorcellement. Il trouve toutefois une solution alternative: "J'y envoie un de nos académiciens habiles qui verra l'ensorcelée, il me fera la relation de tout» (P 87: fo $\left.233 \mathrm{v}^{\circ}\right)$. Le 22 septembre, il annonce:

Mon amy qui est allé à Charenton me doit donner une Relation de son voyage que je feray tenir à V. A. Se. J'ay sceu desja que $\mathrm{M}^{\mathrm{r}}$ de Menars y a esté avec le curé de $S^{t}$ Laurens, syndic du clergé, les curés de $S^{t}$ Eustache et de $S^{t}$ Gervais et l'official de Paris, ce qui a un air juridique et de somation. (P 87: fo $\left.254 \mathrm{v}^{\mathrm{o}}\right)$

Le lendemain, il précise dans une nouvelle lettre qu'il envoie la relation, qui est donc jointe, à part, à son courrier ${ }^{20}$. La curiosité pour les phénomènes étranges et inhabituels est souvent un des traits décrits comme typiques des érudits et férus de sciences de l'époque moderne (Schnapper 1988). Il est toutefois notable que le phénomène ne fasse ici l'objet d'aucun commentaire dissertatif de la part de Bourdelot. Il rapporte simplement l'information au prince de Condé et le prévient qu'il lui transmettra un compte-rendu après être allé sur place. Si l'on fait abstraction du contenu de l'affaire, nous sommes là dans le cadre du service habituel de Bourdelot qui transmet régulièrement des informations de tous types au prince. Il utilise ainsi un dispositif courant dans la transmission épistolaire de nouvelles, notamment militaires, où l'expéditeur mentionne dans sa lettre un événement en précisant qu'il joint un compte-rendu plus précis à part ou bien où il annonce l'envoi d'un document établi par un tiers ${ }^{21}$.

20. La relation n'est toutefois pas conservée avec la lettre de Bourdelot du 23 septembre (P 87: fo 261).

21. On retrouve cela dans de nombreuses lettres de Bourdelot, notamment au début des années 1670 alors que Condé se trouve engagé dans des opérations liées à la guerre de Hollande et est donc fortement en demande de nouvelles 
Nous voyons donc Bourdelot mobiliser son réseau et sa capacité à drainer et à traiter de nombreuses informations dans le cadre de son service auprès du prince. Bourdelot fait en effet partie de ces acteurs rompus aux "traditions of information management" qui se développent à l'époque moderne devant la surcharge d'information suscitée notamment par l'expansion de l'imprimé (Blair 2011: 1-3). Si les techniques d'écriture, d'annotation et de gestion des notes et mémoires n'apparaissent pas dans la correspondance, la spécificité du lien épistolaire entre le médecin et son patron fait que les techniques et usages permettant d'obtenir de l'information sont le plus souvent explicites. En effet, la lettre sert aussi à constamment réactualiser la confiance accordée par le prince à son médecin, confiance en sa fidélité, en son implication et en sa capacité à remplir ses offices. Ainsi, Bourdelot ne se contente pas d'envoyer la relation une fois qu'il la possède; il ne cesse, du 17 au 23 septembre, de tenir Condé au courant de l'avancée de ses manœuvres pour obtenir un compte-rendu précis de l'étrange phénomène par l'entremise de l'un des participants aux réunions de l' "Académie Bourdelot». Le petit cercle apparaît ici comme une réserve de personnes dévouées à Bourdelot et à travers lui au prince de Condé. Le cas n'est pas isolé; par exemple, dans une lettre du 22 décembre 1644, Bourdelot écrit: "On nous dit hier force nouvelles, qui fut jour d'assemblée, que je vous écrirai comme on nous les a dites» (M 31 : fo 411). L'assemblée désignée est encore ici le cercle qui se réunit autour de Bourdelot et qui sert notamment à centraliser informations et nouvelles. Le lien entre traitement de l'information et cercles savants n'est d'ailleurs pas exceptionnel. À propos du savant aixois Nicolas Claude Fabri de Peiresc, Ann Blair explique qu'il "diffused the material in his notes through abundant letter writing, serving as a clearing-house for information for the Republic of Letters" (Blair 2011: 87).

Le cas de Bourdelot et des autres "érudits» présents dans la correspondance du prince de Condé (comme Isaac de Lapeyrère) montre que l'espace de redistribution de l'information et des savoirs qu'ils collectent, échangent et traitent ne se limite pas à des cercles savants, mais irrigue aussi des lieux de pouvoir à travers la correspondance. Bourdelot et ses amis participent donc à un des aspects les plus importants de la pratique politique: le renseignement. Le fait incite à s'interroger de nouveau sur la nature de la curieuse affaire qui est en jeu. Comme on l'a déjà vu, Bourdelot explique d'emblée que " $\mathrm{M}^{\mathrm{r}}$. De la Renye $[s i c] »$ doit le mener à Charenton et mentionne que le roi se préoccupe de la situation. Le 22 septembre, il rapporte la venue de l' « official de Paris ${ }^{22}$ » et souligne la tournure «juridique» que prend l'affaire. Nicolas de la Reynie, que Bourdelot devait accompagner, est alors lieutenant général de police. L'incident est d'emblée associée à un cas de sorcellerie puisque la personne concernée «accuse une vieille femme de luy avoir donné un sort». Or, il s'agit là d'un domaine sensible. En 1682, la cour est encore marquée par la toute récente affaire des poisons sur laquelle le lieutenant général de police fut chargé d'enquêter et dont Bourdelot rapporte tous les échos et rumeurs à Condé dans ses lettres. Et cet épisode associe justement rumeurs de sorcellerie, criminalité et troubles politiques. Bien plus qu'un cas de curiosité pour savants, l'histoire de la petite vomisseuse de crapauds est donc une affaire politique sensible, pouvant engendrer des troubles et qui attire donc à ce titre l'attention du pouvoir. Il est ainsi logique que Condé se tienne informé et que Bourdelot mette tant d'application à le tenir au courant. Finalement, la police conclut à une supercherie et l'affaire se trouve être sans conséquence ${ }^{23}$. Ici encore, l'examen de ce cas montre la porosité et l'étroite proximité entre, d'une part, les pratiques

militaires et diplomatiques. Voir par exemple P 44, fo 257, 258 et 305; P 45, fo 137, 166 et 173.

22. L'official est un clerc chargé par l'évêque de rendre la justice en son nom. Il s'agit donc d'un juge ecclésiastique.

23. Sigaud de La Fond, qui évoque cette affaire à l'article «Estomac» de son Dictionnaire des merveilles de la nature (1781), explique que le lieutenant criminel, Jacques Defita, fit avouer à la jeune femme qu' elle "avaloit, en cachette $\&$ avec un desir singulier, des chenilles, des araignées \& autres insectes » (I: 265-266). 
érudites des curieux qui font leur miel de cas étranges au carrefour de la médecine, de la science et du surnaturel, et, d'autre part, les pratiques politiques, notamment d'information.

Parmi les autres pratiques associées aux échanges savants et érudits, la relation d'expérience tient une place importante. Nous avons analysé l'exemple malheureux de Bernardin Martin. Mais qu'en estil lorsque l'intéressé est un savant notoire ou bien que la manipulation semble à la pointe des découvertes? L'expérience de Torricelli sur le vide, alors bien connue des savants, est ainsi reproduite en 1647 lors d'une séance de l' "académie Bourdelot» et fait l'objet de courts comptes-rendus descriptifs dans les lettres que Bourdelot envoie à certains de ses amis, comme l'érudit Cassiano dal Pozzo (Pintard 1951). Dans la correspondance du Grand Condé, la mention d'expériences est toutefois relativement rare. Elles ne font en tout cas pas l'objet de comptes-rendus précis. On observe ainsi une mention d'expérience dans une lettre adressée par Bourdelot au prince de Condé le 26 septembre 1682. Le médecin y écrit: " $\mathrm{M}^{\mathrm{r}}$ le Sauveur et son élève font tous les matins des expériences hydrauliques à la fontaine du petit bois de l'hostel de Condé» (P 87: fo 274). Joseph Sauveur est un mathématicien, notamment connu pour ses travaux portant sur l'acoustique. Il est recruté par le fils du Grand Condé, Henri Jules, pour être l'un des précepteurs du jeune duc de Bourbon, son fils. Ce que Bourdelot évoque ici, ce sont donc des expériences éducatives visant à donner des notions de science au jeune prince et non des expériences témoignant d'une activité scientifique au sein de la maison Condé. Bourdelot informe régulièrement sur ces matières le grand-père, qui se soucie de l'éducation d'un petit-fils qui ne brille pas autant qu'il l'espérerait et qui échoue d'ailleurs, en 1683, à la traditionnelle «dispute» sanctionnant ses deux années de philosophie au collège de Clermont (Béguin 1999a: 276). Une fois de plus, le savoir est bien présent, mais plutôt sous la forme d'une préoccupation ordinaire dans une maison princière que sous celle d'une singulière activité scientifique autour du prince.

Les lettres impliquant des acteurs dont l'identité est en partie construite sur des connaissances fines dans un ou plusieurs domaines et dont le propos se rapporte à des activités considérées comme caractéristiques des milieux érudits (relations de phénomènes curieux, expériences, etc.) n'apparaissent donc pas dans la correspondance du Grand Condé comme des lettres savantes ou comme des lettresdissertations, si ce n'est par accident. Ce qui est remarquable, c'est qu'elles n'en laissent pas néanmoins d'être fondées sur des usages sociaux des savoirs. Elles appartiennent donc à la fois au domaine savant et à celui de la politique, qui ne peuvent être séparés puisque l'usage des savoirs permet la pratique politique épistolaire, tout comme l'activité épistolaire politique qui se déploie dans la correspondance du prince ne cesse de mobiliser des savoirs spécifiques et les acteurs qui les possèdent.

\section{Isaac de Lapeyrère, homme d'action érudit}

Isaac de Lapeyrère présente le profil du parfait érudit. Fils d'un petit noble protestant, il s'intéresse à la critique biblique et aux questions de théologie. En 1643, il publie Du rappel des Juifs et, en 1655, les Preadamite où il bouleverse la chronologie biblique en affirmant que des hommes auraient préexisté à Adam, qui ne serait, lui, que l'ancêtre des juifs. Il entre au service du prince de Condé dans les années 1640. On le trouve d'ailleurs désigné comme "domestique» dans une lettre de François Blondel adressée au prince le 16 février 1655 (P 15: fo 17). Il semble assurer principalement des fonctions de bibliothécaire, même si ses missions sont extrêmement diversifiées. Au terme de l'année 1655, la publication des Preadamite provoque un scandale et le fait condamner par les autorités ecclésiastiques de Namur. Il reste emprisonné environ sept mois à Bruxelles (Oddos 2012: 75-86; Pietsch 2012: 236238). Auprès du prince, on constate que ses compétences de savant sont effectivement mises à profit, 
mais dans des domaines qui semblent parfois s'éloigner fort de la stricte érudition. Quatre lettres de Lapeyrère écrites au Grand Condé sont conservées. Une lettre écrite le 29 octobre 1667 donne une bonne idée de ce qu'un personnage possédant une solide culture classique pouvait apporter au service d'un prince. Lapeyrère y revient sur la présentation qu'il a faite quelques jours plus tôt au prince d'une devise latine. Il commente la manière dont ce dernier a su marquer, par l'intonation de sa lecture, sa compréhension fine de la devise et par là même de l'intention de son auteur, Lapeyrère:

Je remarquay, Monseigneur, que V. A. $S^{\text {er }}$. appuya sur le mot ruit, de la devise qu'il y a quelques jours, j'eus l'honneur de luy présenter. Je ne pouvois, ce me semble, mieux exprimer l'ardeur avec laquelle

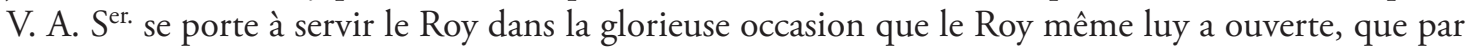
l'impétuosité avec laquelle un torrent se précipite par l'ouverture qu'on luy a faite et avec ce mot: qua data porta, ruit ${ }^{24}$. Ce fut avec la même ardeur que Barac, grand Capitaine et Prince du peuple de Dieu, sortit contre deux Roys ses ennemis, et revint triomphant de leur défaite. L'histoire des Juges dit que les filles juives chantoient ce triomphe. Barac quasi in preceps et barathrum, se discrimini dedit ${ }^{25}$. Hercule se jeta de cette sorte dans la caverne de ce Monstre demy homme et demy bête, qui vomissoit des Tourbillons de feu et de fumée. Ce que Virgile décrit ainsi ${ }^{26}$ [...]. Ça a été, Monseigneur, avec une pareille impétuosité que V. A. S ${ }^{\text {er. }}$ s'est jetée si souvent dans les périls, et dans des tourbillons de feu et de fumée [...]. (P 36: fo 260)

Condé est donc comparé, par son courage et son ardeur au combat, à une figure biblique de chef de guerre libérant le peuple de Dieu, ainsi qu'à Hercule se précipitant pour étrangler un monstre. Le reste de la lettre voit se développer et s'amplifier cet éloge du courage princier. Lapeyrère y évoque les succès de Condé en Allemagne, affirme que le prince a dépassé les héros de l'Antiquité et prophétise, dans le contexte de la guerre de Dévolution, de nouveaux succès pour le glorieux chef de guerre "sans doute animé par de nouveaux transports d'y étendre les limites de l'empire de Notre Grand Roy» (P 36: fo 260). On retrouve ici des traits caractéristiques des usages érudits avec l'insertion des citations latines et la mise en avant d'une connaissance fine de la littérature antique dans le texte. Lapeyrère utilise même sa connaissance approfondie des livres historiques de l'Ancien Testament pour produire un discours élogieux original à la gloire de Condé. La lettre nous permet également de déterminer l'une des fonctions de l'auteur des Preadamite auprès du prince. C'est en effet une pratique commune au XvII ${ }^{\mathrm{e}}$ siècle que de faire appel à des érudits pour rédiger des sentences latines inspirées par la culture classique et destinées à célébrer la personne et les exploits d'un prince ou d'un grand noble à l'occasion d'un événement particulier (Charton 2011). Ici, Lapeyrère s'inspire directement de l'Énéide, où Virgile décrit les vents libérés par Éole s'engouffrant hors de la montagne (chant I, vers 83: qua data porta, ruunt). Ces devises trouvent le plus souvent place sur des médailles ou peuvent éventuellement venir orner des gravures. L'érudit polygraphe François Nicolas Baudot Dubuisson-Aubenay, familier du secrétaire

24. Par la brèche ouverte, il s'engouffre (nous traduisons).

25. Lépisode se situe au Livre des Juges, (V, 15), dans le "Cantique de Débora et Baraq". Il s'agit d'un chant de victoire et d'action de grâces célébrant une victoire des Israélites sur Yabîn, roi de Canaan, qui les opprime. Le cantique exalte les tribus Nephtali et Zabulon qui ont répondu à l'appel de Débora, prophétesse qui rend la justice au nom de Yahvé, et dont les armées sont dirigées par Baraq, fils d'Abinoam. Dans la Vulgate, la citation exacte complète est: Duces Issachar fuere cum Debbora, et Barac vestigia sunt secuti, qui quasi in preceps ac barathrum se discrimini dedit. Lapeyrère restitue donc une citation tronquée, que l'on peut traduire par: Barac se jeta au cour du danger, comme dans un précipice et un abîme.

26. Suit une citation latine scrupuleusement exacte de L'Énéide de Virgile, où Hercule combat le géant Cacus (chant VIII, vers 256 à 258). 
d'État Henri du Plessis-Guénégaud, conçoit par exemple dans les années 1640 des projets de devises en latin pour les jetons du Conseil d'État (Juren 1976).

Une lettre antérieure, datée du 8 octobre 1655 et écrite de Namur, nous donne à voir un autre aspect des relations entre Lapeyrère et le prince de Condé. L'érudit y représente au prince la légitimité qu'il a à prétendre à la fonction occupée par un agent de Condé en Espagne qui vient de décéder, M. de Saint-Agoulin, en insistant sur le mauvais accueil qu'il a lui-même subi alors qu'il avait été envoyé par Condé à Madrid, mauvais accueil qui l'a obligé à rentrer en France. Il cherche donc à obtenir une position qui lui permettrait de revenir triomphant dans la capitale espagnole. En 1655, Condé est en exil dans les Pays-Bas espagnols et occupe des fonctions de commandement à la tête des troupes des Rois Catholiques. Lapeyrère s'emploie alors à développer une théorie de l'importance du renseignement et des stratégies de collecte d'informations. Il explique combien il aurait souhaité pouvoir servir d'espion en étant attaché au comte de Fiesque, alors ambassadeur du Grand Condé à Madrid, et rappelle son expérience dans ce domaine en mettant en avant son habileté:

[...] j'aurois esté si heureux que de servir V. A. Ser ${ }^{m e}$ sous luy, j’aurois pratiqué beaucoup de personnes, qui m'auroient dit une infinité de choses qu'il importe que V. A. Ser ${ }^{\text {me }}$ scache et que l'on ne peut apprendre que par le commerce de toutes sortes d'esprits, et de toutes sortes de conditions. De quoy, il est certain, Monseigneur, que vous auriez reçu, non seulement du plaisir et du divertissement, mais de l'utilité aussy. Car il doit estre des grands Princes, et de ceux principalement qui ont des affaires de l'importance des vostres, ce que les anciens disoient du Soleil, qu'il voyoit et qu'il entendoit tout ${ }^{27}$. Il importeroit, Monseigneur, que V. A. Ser ${ }^{\text {me }}$ put tout voir, pour bien juger de tout. Mais puisque cela ne se peut parce qu'elle ne peut pas estre partout, il est du moins nécessaire qu'elle apprenne par le rapport d'autruy, et qu'elle entende ce qu'elle ne scauroit voir. C'est pour cela que sont establis les ambassadeurs. Et comme ils ne peuvent pas eux mesme ni tout voir ni tout entendre, il faut qu'ils ayent des personnes avec eux, qui voyent et qui entendent pour eux. Or, Monseigneur, je diroy sans vanité, que j'aurois esté fort propre pour cela [...]. (P 15: fo 347)

La lettre semble prendre la tournure d'un petit traité de savoir-faire politique. Lapeyrère utilise sa position de docte connaisseur des sciences politiques en plaçant son discours au rang de vérité générale. Il cite Homère, sans le nommer, probablement afin de créer un jeu de connivence avec le prince à même de situer la référence. La suite de la lettre glisse progressivement vers le particulier et sa personne, en montrant comment son service auprès de Gaspard Coignet de La Thuilerie, ambassadeur aux conférences de Westphalie, lui a permis d'exercer avec succès de telles fonctions d'agent de renseignements. Lapeyrère propose ainsi une réflexion sur la manière d'employer de manière optimale un domestique tel que lui. On saisit alors la richesse et la complexité de la position de ces "érudits» qui vivent des usages sociaux qu'ils font de leurs savoirs, de leurs compétences et des perspectives que leur notoriété de docte leur ouvre. En effet, la proposition de Lapeyrère n'est en rien inédite. Certains passages de son récit sont chiffrés, ce qui montre que Condé l'utilise déjà régulièrement pour se faire transmettre des informations sensibles. Posséder un chiffre permettant de communiquer avec le prince tient en effet du privilège et marque à la fois l'importance de Lapeyrère dans la galaxie Condé, mais aussi le caractère stratégique de sa fonction. On constate ensuite que Lapeyrère fait montre d'une véritable ambition, qui touche non pas à sa reconnaissance publique en tant que savant et lettré, mais à son habileté en matière

27. Il s'agit d'une référence à l'Odyssée (XI, 109) : «Aussitôt qu'échappés à la mer violette, ton solide vaisseau vous mettra sur les bords de l'Ile du Trident, vous trouverez, paissant, les vaches du Soleil et ses grasses brebis: c'est le dieu qui voit tout, le dieu qui tout entend!» (Homère, L’Odyssée: «Poésie homérique», II: 87). 
de renseignement et de diplomatie interlope. Le portrait se complique lorsque l'on prête attention à la thématique du "plaisir et du divertissement» sur laquelle Lapeyrère insiste. Les lettres emplies d'informations ne servent donc pas uniquement à gouverner, mais aussi à distraire. Elles semblent, pour ainsi dire, régies par l'exigence classique du "plaire et instruire», aussi bien que des textes qualifiés a posteriori de littéraires. Le prince s'entoure en fait de gens qui savent bien qu'en matière de service, plaire est instruire et instruire est plaire, ce qui nécessite une habileté et une aisance particulières, aussi bien avec la langue que les savoirs. Cela montre combien les frontières entre érudition, pratique politique, mais aussi activité lettrée sont extrêmement mouvantes et ne doivent pas servir à enfermer des acteurs dans des catégories lorsqu'il s'agit de rendre compte de l'identité et de la fonction de personnages comme Lapeyrère, dont le parcours ne prend sens que si on le replace dans la logique d'une carrière d'homme de savoir intégré dans la société de son temps. Lapeyrère n'est d'ailleurs pas parvenu à travailler au service de Gaspard Coignet de La Thuilerie, puis de Condé, uniquement de par son aura de savant qui aurait incité ces puissants à le fréquenter pour profiter des lumières de son esprit. La famille d'Isaac de Lapeyrère possède d'anciens liens de clientèle avec les Condé (Pietsch 2012: 125). Son père, Bernard, comme plus tard trois de ses frères, est contrôleur des guerres de Guyenne, province dont le gouvernement a été donné en 1596 par Henri IV à Henri II de Bourbon, le père du Grand Condé. Enfin, Jean Daliès, cousin d'Isaac, homme d'affaires au service du prince, gère les biens d'Henri II de Bourbon en Guyenne, notamment le duché d'Albret. On voit ainsi se confirmer ce que Katia Béguin met en lumière lorsqu'elle insiste sur le fait que le patronage est rarement individuel et "qu'il y a là un modèle normatif commun à l'ensemble de la société d'Ancien Régime, qui ne connaît guère que des promotions collectives" (Béguin 1999a: 202). Elle oppose alors ce modèle à celui du mécénat dans lequel la relation est "foncièrement individuelle» (p. 202). Et effectivement, nous sommes bien ici encore face à une relation clientélaire qui prend la forme d'un service domestique. Ce qui est remarquable, c'est que Lapeyrère présente bien sa capacité à servir le prince dans le domaine du renseignement comme le fait non seulement de sa maîtrise fine de l'écrit, qui lui permettrait de produire des comptes-rendus efficaces, ou encore de ses connaissances de diplomatie ou de tactique militaire, mais bien de son érudition en matière de théologie et de sa capacité à user de sa raison dans des discussions savantes. Lapeyrère explique en effet qu’à Madrid, les autres envoyés de Condé n’ont cessé de tenter de le disqualifier aux yeux des Espagnols en l'identifiant comme «huguenot et préadamite»; mais l'effet obtenu n'est pas celui recherché:

[...] cela donnoit la curiosité à beaucoup d'autres de m'accoster et de m'entendre parler, croyans que je fusse quelque animal fantastique. Mais, comme ils voyaient que je raisonnois comme les autres hommes, et que je scavois mesme des choses que les autres qui me vouloient descrier ne scavoient pas, j'acquerois de l'estime parmi eux et y aurois acquis assurément le crédit qu'il faut pour y servir utilement V. A. Ser ${ }^{\mathrm{me}}[\ldots]$. (P15, fo 347)

Le renversement est donc total. Lapeyrère, doublement marginal de par sa religion et ses écrits sulfureux, "animal fantastique» tenu à distance par les agents mêmes de son patron, parvient à se glisser par la qualité de sa conversation dans les réunions de la bonne société espagnole, hors des audiences réglées, dans ces conversations particulières, si communes que la langue se relâche et que les informations s'échappent. Certes, l'hostilité des envoyés de Condé à Madrid finit par le forcer à partir, mais la lettre adressée au prince produit dans son contenu la démonstration orchestrée par Lapeyrère de l'efficacité de l'érudition dans la pratique politique en ce qu'elle fonctionne comme une sorte d'opérateur social permettant au savant d'endosser plusieurs fonctions et de se glisser dans des milieux 
divers, subissant des métamorphoses identitaires, de l' «animal fantastique» au docte à la conversation profitable pour l'esprit. Lapeyrère est tout cela, en même temps, et tel est son outil de travail. Érudit passionné par l'étude, il l'est assurément. Habile diplomate de l'ombre et serviteur des princes, il l'est tout autant. Et jamais l'un sans l'autre. Lapeyrère ajoute à sa lettre un document à part, un mémoire, qui vise à expliquer au prince les raisons de l'hostilité de ses propres envoyés à l'égard de l'auteur des Préadamites. Il la justifie par un épisode militaire survenu en 1653, aux alentours de Bordeaux, alors que le comte de Fiesque tente d'organiser la retraite des derniers Condéens lors de la reddition des Bordelais à la fin de la Fronde. Lapeyrère décrit Fiesque comme un piètre commandant, prenant des décisions inconsidérées et se forgeant une solide rancune contre le huguenot, habile stratège militaire lui soufflant des manœuvres qu'il ne veut pas exécuter. Le mémoire est un formidable récit de bataille, rédigé d'une plume alerte et doublé d'une analyse mettant en valeur le talent d'Isaac de Lapeyrère en matière militaire. Le récit sert bien sûr à ôter tout doute dans l'esprit du prince sur les raisons ayant amené Fiesque et d'autres de ses envoyés en Espagne à s'opposer à Lapeyrère. Il parachève surtout le plaidoyer de Lapeyrère destiné à se tailler une place au cœur de la machine politico-militaire de Condé, en montrant, une fois de plus, son talent en action.

L’observation de la présence érudite dans la correspondance du Grand Condé confirme ainsi ce que plusieurs travaux sur l'épistolarité avaient déjà souligné (Boutier et al. 2009; Beaurepaire et al. 2006): la lettre est un véritable outil pour l'agir politique. L'envisager ainsi permet tout aussi bien de mettre au jour la variété des ressorts stratégiques utilisés dans l'écriture pour tenter de se frayer un chemin dans une société dont sont dévoilés ainsi les règles et les rouages, que d'approcher l'identité des épistoliers dont les actes d'écriture révèlent des identités sociales complexes qui échappent aux catégories traditionnelles. Considérer la présence d'acteurs possédant une légitimité savante permet ainsi de montrer que leur implication dans la vie de la cité n'est en rien marginale et n'est dans tous les cas pas séparable de leurs compétences et de leurs appétences pour des savoirs multiples ou extrêmement spécialisés. L'échange épistolaire construit ainsi un espace de communication dont les règles, qui apparaissent parfois de manière particulièrement nette lorsque se produisent des écarts (comme dans le cas de Bernardin Martin), révèlent la nature de la relation entre le prince et ses domestiques "érudits ». Cette relation n'est fondée ni sur la transmission de savoirs à un prince ami des arts, des lettres et des sciences, ni sur un simple rapport de consommation de compétences et de services. Elle est avant tout le produit d'interactions d'acteurs où un prince du sang, avec tous les devoirs et charges que sa position implique, trouve profit, efficacité et agrément dans la compagnie de domestiques efficaces, possédant des compétences singulières et des savoirs multiples, tandis que ces derniers trouvent matière à employer leurs talents. Les lettres ne sont pas ici des dissertations destinées à une sédimentation des savoirs, elles sont une pratique des savoirs en action.

\section{Références bibliographiques}

Allaire, E. 1886. La Bruyère dans la maison de Condé: Études biographiques et historiques sur la fin du XVIt siècle, vol. 1. Paris: Didot.

Bayle, P. 1697. Dictionnaire historique et critique, 2 vol. Rotterdam: R. Leers.

Beaurepaire, P.-Y., J. Häseler et A. McKenna, dir. 2006. Réseaux de correspondance à l'âge classique (XVI'-XVIII siècle). Saint-Étienne: Publications de l'Université de Saint-Étienne.

Beaurepaire, P.-Y. 2014. "Introduction». Dans La communication en Europe de l'âge classique au siècle des Lumières, sous la direction de P.-Y. Beaurepaire. Paris: Belin : 5-8. 
Béguin, K. 1999a. Les princes de Condé: Rebelles, courtisans et mécènes dans la France du Grand Siècle. Seyssel: Champ Vallon.

Béguin, K. 1999b. "Un mécénat scientifique au Grand Siècle: Condé et l'académie Bourdelot». Dans Mécènes et collectionneurs, vol. I: Les variantes d'une passion, sous la direction de J.-Y. Ribault. Paris: Éditions du Comité des travaux historiques et scientifiques: 81-93.

Blair, A. 2011. Too Much to Know : Managing Scholarly Information before the Modern Age. New Haven : Yale University Press.

Boutier, J., L. Sandro et O. Rouchon. 2009. La politique par correspondance : Les usages politiques de la lettre en Italie (XIV-XVIII siècle). Rennes: Presses universitaires de Rennes.

Brizay, F. 2015. «Introduction. Érudition et culture savante». Dans Érudition et culture savante de l'Antiquité à l'époque moderne, sous la direction de F. Brizay et V. Sarrazin. Rennes: Presses universitaires de Rennes: 7-15.

Cabanis, C. 1987. Les apothicaires et la Réforme: Contribution à l'histoire de la Pharmacie française (XVI ${ }^{e}$-XVIII ${ }^{e}$ siècle. Paris: Fernand Lanore.

Cavaillé, J.-P. 2011. "Le "libertinage érudit": fertilité et limites d'une catégorie historiographique", Les Dossiers $d u$ Grihl, Les dossiers de Jean-Pierre Cavaillé, Libertinage, athéisme, irréligion. Essais et bibliographie, http://journals.openedition.org/dossiersgrihl/4827, consulté le 20 juillet 2018.

Chareix, F. 2006. La philosophie naturelle de Christiaan Huygens. Paris: Vrin.

Charton, F. 2011. Une institution au service du Prince: de la Petite Académie à l'Académie des Inscriptions et BellesLettres [1663-1742]. Thèse de doctorat, École des hautes études en sciences sociales [EHESS].

Cosandey, C. 2016. Le rang: Préséances et hiérarchies dans la France d'Ancien Régime. Paris: Gallimard.

Fumaroli, M. 2015. La République des Lettres. Paris: Gallimard.

Furetière, A. 1690. Dictionnaire universel, contenant generalement tous les mots françois, tant vieux que modernes, et les termes de toutes les sciences et des arts. 2 vol. La Haye et Rotterdam: A. et R. Leers.

Grell, Ch. 1993. L'Histoire entre érudition et philosophie: Étude sur la connaissance historique à l'àge des Lumières. Paris: Presses universitaires de France.

Gysel, C. 1980. «Bernardin Martin et sa "Dissertation sur les Dents" [1679-1979]». Actualités odonto-stomatologiques 129: 59-71.

Häseler, J. et A. McKenna. 2006. «Introduction: De la lettre à la correspondance et du réseau à l'espace social ». Dans Réseaux de correspondance à l'àge classique [XVIe-XVIII siècle], sous la direction de P.-Y. Beaurepaire, J. Häseler et A. McKenna. Saint-Étienne: Publications de l'Université de Saint-Étienne: 7-16.

Homère. 1947. L'Odyssée: «Poésie homérique», 3 vol. Texte établi et traduit par V. Bérard. Paris: Les Belles Lettres. [1924].

Inventaire après décès de Pierre Boulle. 1635. MC/ET/VII/24. Archives nationales: Paris.

Juren, V. 1976. «Les projets de Dubuisson-Aubenay pour les jetons du Conseil d'État ». Revue numismatique 18 : 156-170.

Kriegel, B. 1988. Les historiens et la monarchie. 4 vol. Paris: Presses universitaires de France.

Lafont, O. 2008. Échevins \& apothicaires sous Louis XIV: La vie de Matthieu-François Geoffroy, bourgeois de Paris. Paris: Pharmathèmes.

Lafont, O. et C. Van Robaeys. 1992. «La pratique de l'art de l'apothicaire au XVII siècle: information fournie par deux inventaires après décès". Revue d'histoire de la pharmacie 295 : 453-466.

Lunel, A. 2008. La maison médicale du roi XVI'-XVIII siècles: Le pouvoir royal et les professions de santé (médecins, chirurgiens, apothicaires). Seyssel: Champ Vallon.

M 31. 1644. Archives du Musée Condé: Chantilly.

Malebranche, N. de. 1961. CEuvres complètes: Tome XVIII. Correspondance et actes 1638-1689. Édition préparée par André Robinet. Paris: Vrin.

Martin, B. 1684. Traité de l'usage du lait. Paris: E. Couterot. 
Moreri, L. 1718. Le grand dictionnaire historique ou le mélange curieux de l'histoire sacrée et profane, 5 vol. Paris: J.-B. Coignard. [1674].

Oddos, J.-P. 2012. Isaac de Lapeyrère [1596-1676]: Un intellectuel sur les routes du monde. Paris: Honoré Champion.

P 15. 1655. Archives du Musée Condé: Chantilly.

P 22. 1659. Archives du Musée Condé: Chantilly.

P 29. 1 663. Archives du Musée Condé: Chantilly.

P 33. 1665. Archives du Musée Condé: Chantilly.

P 36. 1667. Archives du Musée Condé: Chantilly.

P 44. 1670. Archives du Musée Condé: Chantilly.

P 45. 1670. Archives du Musée Condé: Chantilly.

P 47. 1673. Archives du Musée Condé: Chantilly.

P 73. 1679. Archives du Musée Condé: Chantilly.

P 81. 1681. Archives du Musée Condé: Chantilly.

P 87. 1 682. Archives du Musée Condé: Chantilly.

P 88. 1682. Archives du Musée Condé: Chantilly.

Panckoucke, C.-L.-F. 1824. Dictionnaire des sciences médicales: Biographie médicale, vol. 6. Paris: Imprimerie de C.-L.-F. Panckoucke.

Pietsch, A. 2012. Isaac La Peyrère: Bibelkritik, Philosemitismus und Patronage in der Gelehrtenrepublik des 17. Jahrhunderts. Berlin: De Gruyter.

Pintard, R. 1943. Le libertinage érudit dans la première moitié du XVII siècle. Paris: Boivin.

Pintard, R. 1951. "Autour de Pascal: l'Académie Bourdelot et le problème du vide». Dans Mélanges d'histoire littéraire offerts à Daniel Mornet, professeur honoraire à la Sorbonne, par ses anciens collègues et ses disciples français. Paris: Nizet: 73-81.

Pujol, S. 2005. Le dialogue d'idées au dix-huitième siècle. Oxford: Voltaire Foundation.

Richelet, P. 1680. Dictionnaire françois, contenant les mots et les choses, plusieurs nouvelles remarques sur la langue françoise... Genève: J. H. Widerhold.

Roulier, D. 2019. «Apparition de la figure de l'Érudit pendant la querelle des Anciens et des Modernes». Dans Reconnaître l'érudition. Actes de la journée d'études des doctorants du CSLF, sous la direction de F. Kerautret, H. Parent, L. Roudier et D. Roulier. Paris: La Taupe Médite: 57-76.

Ruel-Kellermann, M. 2010. "L'héritage castillan de Francisco Martínez de Castrillo. De Bernardin Martin à Pierre Fauchard", Actes. Société française d'histoire de l'art dentaire 15: 42-46. http://www.biusante.parisdescartes.fr/sfhad/actes_2010.htm, consulté le 20 juin 2019.

Schapira, N. 2003. Un professionnel des lettres au XVII siècle: Valentin Conrart: une histoire sociale. Seyssel: Champ Vallon.

Schlanger, J. 1994. «L'érudition et ses ennemis». Poétique 99: 277-289.

Schnapper, A. 1988. Le géant, la licorne et la tulipe: Collections et collectionneurs dans la France du XVII siècle. Paris: Flammarion.

Sigaud de La Fond, J.-A. 1781. Dictionnaire des merveilles de la nature, 2 vol. Paris: Rue et Hôtel Serpente.

Viala, A. 1985. Naissance de l'écrivain: Sociologie de la littérature à l'âge classique. Paris: Les Éditions de Minuit. 